\title{
Science-fiction et science : vie extraterrestre
}

\section{Science fiction and science about extraterrestrial life?}

\author{
Jean-Claude Serge Lévy ${ }^{1}$ \\ ${ }^{1}$ Professeur émérite à l'Université de Paris ${ }^{1}$
}

RÉSUMÉ. La question de la possibilité d'une vie extraterrestre est aussi ancienne que l'observation de la nuit étoilée. Cette question a permis à tout un art de se déployer et a conduit à la science-fiction. L'apport récent des sciences mathématiques et physiques à l'étude du vivant donne l'espoir de perspectives générales et donc la possibilité d'étude de vie extraterrestre. La diversité physique de l'espace extraterrestre y propose de nouvelles formes et donc de nouvelles formes de vie. Enfin les risques de crise sur Terre accentuent l'intérêt porté à la vie extraterrestre.

ABSTRACT. The question of an extraterrestrial life is as old as stars in the night. A whole art has been devoted to this question. The recent involvement of mathematics and physics in life sciences brings the hope of a general view of life in space. The space diversity must lead to other life ways and forms. Finally possible terrestrial crises increase the interest in extraterrestrial life.

MOTS-CLÉS. vie extraterrestre, science-fiction, morphogénèse, formes vivantes.

KEYWORDS. extraterrestrial life, growth and form, living forms.

L'article introductif de Georges Chapouthier ${ }^{2}$ montre les problèmes posés par la possibilité de vie extraterrestre pour notre imaginaire. Le succès permanent de la science-fiction et son rapport à la connaissance scientifique actuelle et en devenir sont des questions fondamentales. L'état de l'art, c'est-à-dire ici de la science, ou plutôt les lignes directrices scientifiques actuellement développées sur ces conditions de vie, encore hors de notre portée, définissent une série de questionnements sur les perspectives de la science-fiction. C'est donc à une double réflexion sur le plaisir de la sciencefiction et sur les contraintes d'un réalisme scientifique que cet article nous mène.

\section{Le plaisir procuré par la science-fiction}

Le plaisir de la science-fiction tient à beaucoup de facteurs plus ou moins explicites. Ainsi, l'omniprésence de la nuit étoilée a toujours interpellé l'humanité. Pour donner une date, on peut citer les calculs des pythagoriciens pour ajuster certains alignements dans les temples égyptiens. Ce souci témoigne d'un profond désir d'intégration de l'humanité dans le cosmos. Ce ciel nocturne troublant nous incite aussi au rêve, ce que la science-fiction concrétise. Comme dans le rêve, la science-fiction reconstruit notre réalité quotidienne en prenant quelque distance, quelque fantaisie avec l'observation. Cette distance, poétique, source de liberté et d'imaginaire, avec, au temps du «siècle des lumières », «les lettres persanes ${ }^{3}$ », un exemple d'une telle distanciation, avait ainsi permis d'introduire une critique subtile de la société de son temps. Plus récemment «1984 $»$ a anticipé les risques d'une future société dominatrice. Cette fantaisie de la science-fiction permet de donner dans «Brave new world ${ }^{5}$ » la présence de parfums, d'odeurs, dans un spectacle à venir, en

\footnotetext{
${ }^{1}$ J.-C.S. Lévy est physicien et anime aussi depuis 2010 des réunions pluridisciplinaires sur le thème « Complexité-désordre ».

${ }^{2}$ G. Chapouthier « De la science à l'art : un imaginaire extraterrestre » ISTE London 2021

${ }^{3}$ C. de Montesquieu « Lettres persanes » Amsterdam 1721

${ }^{4}$ G. Orwell « 1984 » paru en français chez Gallimard Paris 1950

${ }^{5}$ A. Huxley « Brave New world" Chatto et Windus Londres 1932 traduit en français : « Le meilleur des mondes » Plon, Paris 1932 
un art encore plus synthétique que ne le sont actuellement l'opéra ou le cinéma ! Cette grande liberté de l'imagination, en restant cependant proche du réel, du quotidien, fait toujours le succès de la science-fiction. A l'heure où quantité d'observations et même d'actions dans l'espace extraterrestre se réalisent et sont projetées, ce succès ne peut pas faiblir.

\section{Science-fiction et science, une voie commune?}

Une grande question se pose alors pour la science-fiction : doit-elle ou non tenir compte des avancées récentes de la connaissance scientifique ? Bien sûr, une telle option n'est pas une nécessité. Mais la science-fiction peut gagner à un tel jeu basé sur un imaginaire plausible, convaincant. Les observations et les réflexions scientifiques récentes peuvent nourrir notre imaginaire en lui donnant un support plus précis. C'est ce défi scientifique que propose Georges Chapouthier et son univers mosaïque ${ }^{6}$. Nous allons essayer de lui emboîter le pas en essayant de préciser quelques contraintes sur les formes possibles de vie extra-terrestre et ainsi de dégager quelques voies d'approche, quelques points de vue nouveaux pour la science-fiction.

\section{La naissance des formes}

Un grand pas dans la révolution Darwinienne a été franchi par d'Arcy Thompson ${ }^{7}$ en introduisant la notion de morphogénèse, la création de formes dans la nature. Les mathématiciens et, René Thom $^{8}$ en particulier, se sont vite attaqués à ce problème en cherchant à mettre en évidence les formes essentielles de la nature. Ces formes géométriques, des singularités, que René Thom a étudiées, sont liées à la mise en équation des systèmes dynamiques. Ses résultats ont même inspiré le célèbre peintre surréaliste Salvador Dali, alors déjà atteint par la maladie de Parkinson, une terrible catastrophe pour ce créateur si habile. Ainsi Salvador Dali a signé en 1983, sur ce thème scientifique abstrait, sa dernière œuvre : «la queue d'aronde- série des catastrophes » ${ }^{9}$, une œuvre dédiée aux formes géométriques mises en évidence par René Thom. Ainsi, tout en subissant le scénario catastrophe imposé par la maladie, le peintre a intégré la science et la science-fiction dans l'art ! Quel message !

\section{Une vision artistique}

Le poète surréaliste Paul Eluard avait déjà anticipé l'observation pure en écrivant plus tôt «la terre est bleue comme une orange ${ }^{10} »$. L'art est aussi une représentation du monde, une représentation plus libre que celle de la science. Et l'art s'épanouit dans le contexte de la société ${ }^{11}$ en mouvement, en évolution. Son développement sait justement intégrer les leçons de la science et de la technique en un aspect directement sensible.

\footnotetext{
${ }^{6}$ G. Chapouthier, « L'homme, ce singe en mosaïque », Préface de Patrick Blandin, Éditions Odile Jacob, Paris, 2001

${ }^{7}$ G. D’Arcy Wentworth Thompson, « On growth and form » Cambridge University 1917, texte français, Forme et croissance, Seuil, Paris, 2009

${ }^{8}$ R. Thom « Stabilité structurelle et morphogénèse » InterEditions Paris 1972

${ }^{9}$ S. Dali (1904-1989) œuvre produite en mai 1983.

${ }^{10}$ P. Eluard, « L'amour, la poésie » recueil de poèmes paru en 1929 et diffusé actuellement par Gallimard Paris

${ }^{11}$ P. Bourdieu, « La distinction, critique sociale du jugement » Les éditions de minuit Paris 1979
} 
Une première piste à suivre pour la science-fiction est donc l'évolution récente de l'approche scientifique de la morphogénèse. La cellule animale ou végétale est un des éléments de base de la mosaïque vivante. Le point de vue de physicien moderne impliqué dans la biologie s'intéresse donc à l'élasticité des cellules ${ }^{12}$, leur mécanique. Cette élasticité des cellules tient essentiellement à celle des membranes et des parois qui les entourent, les limitent, les protègent. Ces récentes études permettent de comprendre la structuration statique d'un ensemble de cellules différentes, leur arrangement en un être vivant.

Le module de cisaillement des cellules, leur autre aspect mécanique, conditionne leur comportement viscoélastique. Ce comportement est, lui, sensible au caractère très inhomogène du contenu des cellules. Ce contenu peut se déformer sous la contrainte appliquée. Il varie donc au cours du temps, se reconstruit et force souvent la présence de lignes de singularités. Ainsi de nouveaux arrangements apparaissent dans les cellules contraintes. Donc les propriétés d'écoulement, de mécanique des fluides ${ }^{13}$ des cellules sont importantes, cruciales pour les formes qui apparaissent alors. Tout un champ d'études nouveau surgit de cette physique biologique.

\section{Début d'organisation}

La division des cellules depuis la cellule initiale, l'embryon, l'embryogénèse, est évidemment un autre élément essentiel de la constitution de cette mosaïque qu'est l'être vivant. Cette étude, déjà entreprise, a apporté quantité de résultats passionnants sur le développement initial de fonctions biologiques très tôt différenciées ${ }^{14}$ autour d'un axe, en quelque sorte une colonne vertébrale, avec une binarité marquée qui permet à l'être vivant de s'adapter à la saisie de l'espace, et de s'y mouvoir. C'est donc tout un champ d'exploration et de modélisation du vivant qui s'ouvre ainsi, avec déjà des résultats significatifs. Evidemment une telle étude de toutes les plantes et de tous les animaux terrestres est loin d'être achevée, mais l'extrapolation peut déjà commencer et même viser des organismes vivants extraterrestres.

\section{La physique de l'espace extraterrestre}

Une question que l'on peut se poser est alors : «Est-ce que les formes de la vie sont partout les mêmes dans l'espace extraterrestre ? ». Une première réponse apportée par l'absence de pesanteur, de gravité dans les déplacements loin de la terre ${ }^{15}$, est évidemment non. Sur les planètes ou les astres, la pesanteur est aussi différente de celle observée sur Terre. Dans les cas de faible pesanteur, une expansion à trois dimensions équivalentes, comme la boule de liquide des astronautes le montre bien, est évidente. Le corps des astronautes qui restent longtemps en apesanteur est lui-même soumis à une telle dilatation. On s'attend donc dans le cas d'une faible pesanteur à des êtres vivants larges et sans réelle direction privilégiée, des sortes de grande boule. Au contraire dans le cas d'une forte pesanteur, sur une grande planète solide par exemple, on s'attend à un écrasement, à une vie limitée à la surface de la planète ou de l'astre ! En d'autres termes on s'attend alors à des êtres plats

\footnotetext{
${ }^{12}$ Un exemple d'une telle recherche : « Comparative study of cell mechanics methods » par. P.-H. Wu, D. R.-B. Aroush, A. Asnacios, et al. in Nat. Methods 15, 491-498 (2018).

13 “Local viscoelasticity of living cells measured by rotational magnetic spectroscopy" J.-F. Berret in Nat. Com. 7 10134 (2016).

${ }^{14}$ Vincent Fleury, La physique de l'embryon, reportage CNRS 2019.

${ }^{15}$ Kitsou Dubois, «Corps modifié en microgravité, complexité et paradoxe du corps en suspens » in « L'avenir de la complexité et du désordre », pp 17-21 éditeurs J.-C.S. Lévy et S. Ofman, Editions Matériologiques, Paris 2018
} 
ou presque ! Cette géométrie nouvelle doit modifier tous les comportements connus sur Terre et par exemple la mobilité. Cela semble très suggestif pour la science-fiction et pour le dessin.

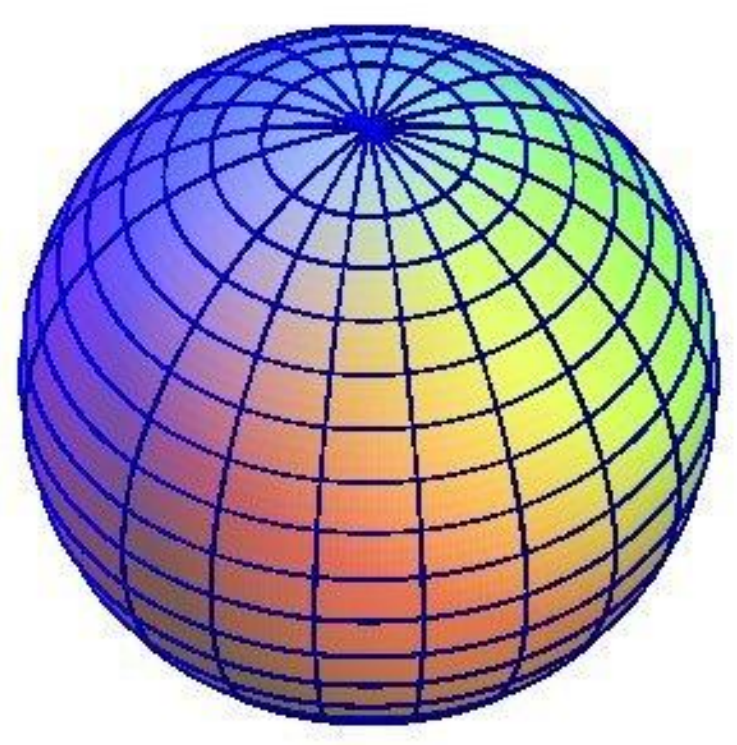

Cellule sphérique dans un espace sans gravité

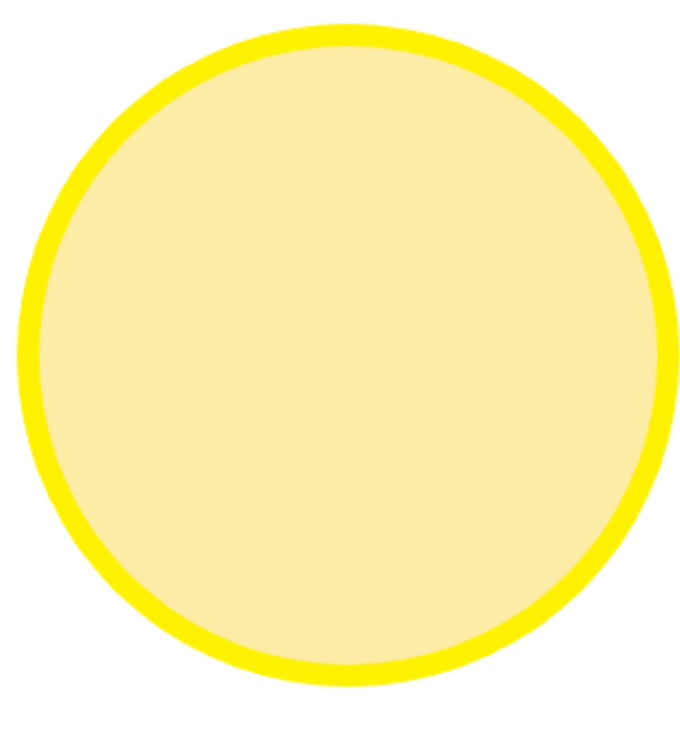

Cellule disque plat en forte gravité

\section{Le déroulement du temps et les accidents de l'histoire}

Enfin, il faut ajouter que la quatrième dimension, le temps, ne s'écoule pas vraiment uniformément, régulièrement, du fait de la complexité de la nature et des «accidents » toujours possibles. Ainsi la disparition des dinosaures, probablement due à une catastrophe climatique, a considérablement modifié la vie sur Terre, en favorisant le développement de formes de vie plus petites, plus adaptables, comme celle de l'Homme. De telles catastrophes peuvent encore se produire. La pollution croissante, liée à une présence humaine toujours plus active et plus nombreuse nous prédit déjà l'émergence de catastrophes climatiques à court terme ! La pandémie actuelle fait aussi craindre l'émergence d'autres pandémies. La question de l'adaptation de l'humanité dans son ensemble à la gestion de tels risques se pose donc intensément. L'échappée dans l'espace apparaît même à certains comme une solution possible, voire nécessaire, à la limite de la science-fiction. Autrement dit, la vie extraterrestre est plus préoccupante que jamais, à différents titres. 